\title{
La ciudad y sus partes: una historia de la institucionalidad local en la Ciudad de Buenos Aires
}

Matías Landau. Universidad de Buenos Aires, Buenos Aires, Argentina.

RESUMEN | Entre fines del siglo xix y fines del siglo xx se han sucedido en la Ciudad de Buenos Aires diversas formas de institucionalidad local. Leemos este proceso como la particular construcción de una relación de gobierno entre los poderes públicos y los habitantes, en la cual el marco de referencia no es la totalidad de la ciudad, sino sus partes constitutivas, esto es, zonas o barrios. El recorrido por diversos momentos históricos (la federalización de la ciudad, las primeras décadas del siglo xx, el peronismo, los años de su proscripción y la era democrática inaugurada en 1983) permite interpretar las continuidades y rupturas de las formas en que se estructuró esta relación. Con ello queremos contribuir a la comprensión de los diversos modos en que se van estableciendo las relaciones de cercanía o lejanía, tanto territorial como simbólica, entre autoridades públicas y habitantes de la ciudad.

PALABRAS ClAVE | gobierno local, historia urbana, participación ciudadana.

ABSTRACT | Between late nineteenth and late twentieth centuries various forms of local institutional arrangements have succeeded one another in Buenos Aires. These local arrangements are seen here as the construction of a government relationship between public authorities and the people, whose frame of reference is not the city as a whole, but its constituent parts, such as zones or districts (barrios). By looking at various historical moments - the federalization of the city, the first decades of the twentieth century, Peronism, the years of its proscription and the democratic era inaugurated in 1983-we seek to interpret the continuities and ruptures of the ways in which this relationship was structured. Our aim is to add to the understanding of the various forms in which closeness and distance, both territorial and symbolic, were established between public authorities and the inhabitants of the city.

KEY WORDS | local government, urban history, citizen participation.

Recibido el 22 de mayo de 2012, aprobado el 3 de noviembre de 2012

E-mail: matiaslandau@hotmail.com 


\section{Introducción}

Este artículo propone un recorrido sociohistórico por las formas cambiantes en que, en diferentes períodos, las elites gobernantes concibieron el modo en que el gobierno de la Ciudad de Buenos Aires debía desarrollar un tipo específico de institucionalidad local. Entendemos por ello la construcción de una relación de gobierno entre los poderes públicos y los habitantes, cuyo marco de referencia no está definido por la totalidad de la ciudad, sino por sus partes constitutivas, esto es, zonas o barrios $^{1}$. El desarrollo de la institucionalidad local puede establecerse siguiendo la instauración de autoridades electivas o la construcción de formas de reconocimiento estatal respecto de organizaciones o instituciones barriales a las que se les asigna una función gubernamental.

El análisis se establece en torno a dos ejes articulados. Por un lado, se examinan las concepciones predominantes, en cada etapa histórica, en relación con los criterios fundamentales sobre los que debe estructurarse el gobierno de la ciudad: si las autoridades deben ser electivas o no, si el voto debe o no ser universal, si las instituciones municipales son meramente administrativas, si es preciso apelar a un saber técnico especializado, etcétera. Por el otro, se indaga en la forma como se vincula cada concepción del gobierno con un modo específico de incorporar la institucionalidad local.

El artículo está dividido en seis apartados. En los cinco primeros realizamos un recorrido sociohistórico, identificando las particularidades de diversos períodos de la ciudad: la situación posterior a la federalización de 1880, la Buenos Aires de entreguerras, el primer peronismo, los ańos que separan la caída de Perón y la dictadura militar de 1976, y aquellos que van desde el retorno democrático a nuestros días. Luego, a partir de lo analizado, realizamos una reflexión que busca plantear las continuidades y rupturas históricas, abriendo un camino para establecer interrogantes sobre el nuevo escenario porteńo, marcado por la puesta en funcionamiento de las comunas.

\section{Buenos Aires luego de la federalización}

Luego de que la Ciudad de Buenos Aires fuera federalizada, en 1880, el Congreso de la Nación se abocó a la discusión de las normas jurídicas que regirían su gobierno. Entre los años 1881 y 1882, los parlamentarios debatieron y sancionaron la ley orgánica de la Municipalidad de la Ciudad de Buenos Aires (Ley 1260)². Los argumentos predominantes se apoyaban en una visión muy frecuente en el pensamiento municipalista decimonónico, que concebía la municipalidad como una agrupación de individuos con intereses civiles en común, dados por sus relaciones

1 El artículo recupera y profundiza uno de los ejes de mi tesis de doctorado sobre las transformaciones históricas en los modos de concebir el gobierno de la ciudad en Buenos Aires durante el siglo xx (Landau, 2010).

2 Sobre el proceso de federalización de la Ciudad de Buenos Aires, véanse Carranza (1926), Pírez (1996), Ruiz (1986), Sábato (2008). Una recopilación de los debates parlamentarios puede consultarse en Jurado (1935). 
de vecindad y sus vínculos económicos ${ }^{3}$. No todos los habitantes de la ciudad eran considerados miembros de la municipalidad, sino solo los "vecinos", que en el lenguaje de la época refería solo a aquellos que contribuían con la ciudad a partir de la paga de un impuesto directo.

De esta particular concepción derivaba la manera de pensar del gobierno de la ciudad, que estaría compuesto por una sumatoria de tareas administrativas de bajo nivel de complejidad. Las tareas "sencillas" del gobierno comunal llevaban a muchos diputados y senadores a sugerir la conveniencia de instituir un cuerpo colegiado en lugar de uno unipersonal, aunque finalmente la ley sancionada estableció la coexistencia de una rama legislativa elegida a través del sufragio (el Concejo Deliberante) y una rama ejecutiva unipersonal (el intendente), designada directamente por el presidente de la Nación. En esta manera de concebir el gobierno de la ciudad solo se estipulaba una participación ligada al sufragio municipal, que era el modo a través del cual los vecinos tomaban parte de su propio gobierno. Pero en tanto que el criterio de pertenencia a la municipalidad se asociaba a la contribución monetaria, el voto no era universal, sino restringido. La ley orgánica municipal impuso como criterio que los individuos que podían ocupar una banca debían ser letrados, mayores y debían pagar un impuesto directo al Municipio, un impuesto comercial o industrial, o ejercer una profesión liberal. Los extranjeros podían, por su parte, ser elegidos si pagaban un impuesto superior a cien pesos. Se imponían algunas condiciones aún más estrictas a los electores, que excluían a los estratos sociales más pobres. Como muestra Guy Bourdé (1974), "en 1890, por ejemplo, para una población de cerca de 500.000 habitantes, se inscriben en las listas 6.754 electores, y solo se vota a 4.034 individuos, lo que representa un 0,01 por ciento de la población!” (p. 99).

Esta particular mirada comenzó a ser modificada a partir de un proceso de crecimiento urbano y de complejización social que significó un acelerado cambio de la ciudad entre las últimas décadas del siglo XIX y las primeras del Xx (Gorelik, 2004; Gorelik \& Silvestre 1991; Hardoy \& Gutman, 1992; Landau, 2011; Scobie, 1977). El crecimiento vertiginoso de la Buenos Aires finisecular fue acompañado de una acción pública, llevada a cabo por el intendente Alvear y sus sucesores, que se enmarcaba en los principios modernizadores que había seguido Haussman en París. En este sentido, desde el poder ejecutivo de la Municipalidad comenzó a desplegarse una racionalidad del gobierno de la ciudad que se alejaba de una concepción ligada a las tareas administrativas sencillas. En su lugar, se planteaba una complejización de las prácticas administrativas, a partir de la invocación de la necesidad de construir un aparato burocrático estatal y del incipiente despliegue de un ideal técnico, ligado a la invocación del saber científico y experto como modo de resolución de los problemas urbanos.

Si bien el programa modernizador en la Buenos Aires finisecular era muy intenso e incluía la apertura de numerosas oficinas públicas y el desarrollo de los servicios urbanos (Pírez, 1998), la rapidez de las transformaciones sociourbanas llevaba a que la presencia del Estado en los sectores más alejados del centro fuera insuficiente. El 
resultado era una gran deficiencia en la infraestructura más básica. La ausencia del Estado llevó a que, en muchos casos, fueran los mismos habitantes del lugar los que comenzaran las tareas de urbanización de los nuevos vecindarios, como las obras de pavimentación de las calles (Tella, 1994). Esta autogestión, lejos de ser criticada, era alabada por parte importante de la opinión pública, que la comenzaba a ver como una forma de colaboración con las autoridades municipales. La Revista Municipal festejaba en 1895 la creación de las primeras "asociaciones de fomento" en los vecindarios recientemente constituidos:

\begin{abstract}
En los actos de la Intendencia, la mayoría de la población solo ve aquellos que se prestan a la crítica (...) solo ve los baches en los afirmados, los depósitos de basuras, los terrenos baldíos, y hasta se responsabiliza al Intendente de las compadradas de los mayorales de tramways ó de los abusos de los inspectores. Y hay que contar que esos mismos que censuran y gritan a tort et a travers invocando a cada rato su título de contribuyente y su amor por el adelanto de la ciudad nada hacen para cooperar à la acción municipal y facilitar su tarea (...). Hay un remedio eficaz para poner término a esta situación inconveniente, retardataria e injusta. Ese medio es la formación de sociedades parroquiales de fomento, que acostumbrando a los contribuyentes a ocuparse de las cosas e intereses municipales serían auxiliares de la autoridad y estímulo a sus esfuerzos (Revista Municipal, 15 de junio de 1895).
\end{abstract}

La cita expresa una crítica a la sola invocación del "título de contribuyente" para lograr un gobierno eficaz. En contraposición, aparece por primera vez una voz que apela a la organización de los habitantes de una zona de la ciudad para colaborar con los poderes municipales. Por supuesto que no hay que sobrevalorar el modo en que por entonces se apelaba a la incorporación de los habitantes en la resolución de las problemáticas de su zona de residencia, puesto que la misma era opacada por la sobrevivencia de un modo de estructurar la relación pública-privada dada por la existencia de las comisiones de higiene, que era la institucionalidad local hegemónica por entonces y que seguía los principales preceptos higienistas, los cuales no solo tenían una gran difusión en Buenos Aires (Armus, 1999; Paiva, 2000), sino que también se extendían a todas las grandes urbes del mundo capitalista. Estas comisiones estaban constituidas por un grupo de "vecinos notables", que eran nombrados directamente por el poder municipal y operaban como delegados administrativos. Su carácter higienista y disciplinario marcaba su objetivo fundamental: controlar el modo de vida de las clases populares.

\title{
Buenos Aires durante los ańos de entreguerras
}

Las primeras décadas del siglo xx fueron testigo de una rápida transformación de la urbe. Si hacia fines del siglo xix todavía la jurisdicción capitalina albergaba el núcleo urbano primitivo, al que se sumaba una cantidad de otros pequeńos núcleos separados por grandes extensiones de campo, las décadas de los años veinte y treinta vieron consolidarse la trama que se extendió a cada rincón de la ciudad, luego de la ampliación de los límites jurisdiccionales sancionados en 1887 (Landau, 2011). El crecimiento de la ciudad fue acompañado de una mayor complejidad económica, 
que redundó en la emergencia de una concepción social de la ciudad (Landau, 2012). Asimismo, el siglo xx argentino trajo consigo una progresiva decadencia del régimen oligárquico, que condujo -luego de varias manifestaciones del conflicto político y social- a la sanción del voto universal, secreto y obligatorio para elecciones nacionales en 1912, inaugurándose así la era de la política moderna con la elección de Hipólito Yrigoyen en 1916. En consonancia con estos cambios, el voto restringido que regía para las elecciones municipales de Buenos Aires comenzó a ser recurrentemente discutido. El proceso local, no obstante, no fue una simple consecuencia directa o una adaptación a la ley nacional. Más bien, se trató de una conjunción de dos procesos que, si bien caminaban de la mano, tenían cierta independencia relativa. En lo nacional no existía el voto censitario y el problema que se debía resolver era el fraude sistemático existente hasta entonces. De allí la necesidad de instaurar el voto secreto y obligatorio. En lo municipal, por el contrario, el voto estaba restringido a unos poquísimos electores y el desafío era lograr una ampliación del padrón electoral.

El nuevo clima de la época llevó a que los principios de gobierno herederos del municipalismo decimonónico, basados en un voto censitario y en un pensamiento administrativo de bajo nivel de complejidad, fueran criticados por aquellos que, como los socialistas, sostenían que Buenos Aires ya no podía ser pensada como una aglomeración de vecinos. Ante la nueva situación, muchos hombres ilustres del momento planteaban la necesidad de reformar el gobierno de la ciudad. Algunos abogaban por la universalización del sufragio. El argumento más utilizado -especialmente por los socialistas, quienes eran los mayores impulsores de esta idea- era que el voto censitario podía haber respondido a los rasgos propios del siglo XIX, cuando Buenos Aires era aún una aldea de pocos habitantes, pero que no se correspondía con la realidad del siglo xx. En 1918 la ley orgánica municipal fue modificada, introduciendo el voto universal masculino para la elección de concejales, aunque manteniendo al intendente nombrado por el presidente de la Nación, y no por sufragio universal como pretendían los socialistas (De Privitellio, 2006).

La otra forma de argumentar contra los principios del gobierno decimonónico se asociaba al desarrollo de un discurso técnico, que se profundizó en las primeras décadas del siglo con el desarrollo de diversos saberes "expertos" en el gobierno de la ciudad, como el del derecho municipal, el de las ciencias de la administración y el del urbanismo. Este tipo de discurso contribuyó a establecer un argumento, muy difundido en las décadas de los veinte y treinta por las elites ilustradas porteñas, que postulaba la necesidad de readecuar el gobierno de la ciudad a las necesidades de una "ciudad moderna" (Landau, 2010). Para ello, se subrayaba el carácter científico-técnico del gobierno de la ciudad, que debía ser cada vez más una tarea de especialistas. La apelación a este ideal técnico permitía, en muchos casos, argumentar por qué el intendente debía ser un ejecutor técnicamente idóneo, más que un representante directo del pueblo.

Es sobre esta tecnificación del gobierno que se planteó la recodificación de la institucionalidad local, más que sobre la universalización del sufragio. El voto, aun universalizado para la población masculina, seguía siendo parcial, puesto que solo se desarrollaba en la rama legislativa, y la elección tenía como referencia la ciudad 
toda y no cada parte. Pero cuando el gobierno comenzó a pensarse cada vez más como el medio de resolución técnica de problemas urbanos, la realidad porteña lo llevó a buscar el medio para institucionalizar la participación de los vecinos. Como expresamos en el apartado anterior, a fines del siglo XIX coexistían dos formas diferentes de institucionalidad local. Una, las Comisiones de Higiene, ligadas a una concepción restrictiva, higienista y disciplinaria, que estructuraba una vigilancia tutelar de los sectores populares por parte de los "vecinos honorables". La otra, las primeras Sociedades de Fomento, que se crearon en diversos vecindarios y que permitían una participación más abierta a los habitantes de la zona. El ocaso del régimen oligárquico y los principios de gobierno del municipalismo decimonónico volvieron anacrónicas las formas adoptadas por las comisiones de higiene, a la vez que fortalecieron las lógicas de las sociedades de fomento.

Fue entonces como parte de ese proceso que, en las décadas de los veinte y treinta, la respuesta municipal vino de la mano de una política que institucionalizó el rol de las sociedades de fomento ${ }^{4}$. De este modo, el gobierno comenzó a tener, por primera vez, un objetivo específico ligado al barrio. Como ha analizado Adrián Gorelik (1994), el barrio no es sinónimo de vecindario, puesto que no se asocia solo al crecimiento de la ciudad, sino que adquiere un nuevo carácter hacia la década de 1910, cuando el Estado empieza a actuar activamente sobre los espacios que hasta entonces tenían escasa relación entre sí. Este vínculo se basó, principalmente, en la relación establecida entre el barrio y los poderes públicos a través del modelo de las sociedades de fomento. En las décadas de los veinte y treinta fueron parte constitutiva de un tipo de gobierno específico que podríamos definir como el "gobierno de los barrios", con la doble lectura que posibilita esta fórmula. Es, en primer lugar, el modo en que los barrios se constituyen como sujetos de gobierno, con sus representantes, sus identidades, sus instituciones. Pero es, también, el modo en que el Estado municipal gobierna los barrios, los construye como objetos de gobierno.

Para comprender este proceso, debemos recordar el modo de relación establecido entre las sociedades de fomento y los poderes públicos. El primer reconocimiento estatal fue el 25 de noviembre de 1919 por medio de una ordenanza sancionada por el Concejo Deliberante. En 1920, el intendente Cantilo creó un registro de las existentes. En 1927, una ordenanza reconocía la exclusividad de la representatividad de cada asociación en un radio delimitado. En 1933, el intendente indicaba que las sociedades de fomento son "entidades representativas" que persiguen el "bien común" del barrio, y creaba una oficina destinada a controlar el vínculo entre el Estado y las asociaciones. En suma, las entidades de este tipo eran especialmente consideradas por la Municipalidad, como se expresa en la Memoria Municipal de 1933-1934, en la que se decía que:

La organización local del vecindario en entidades populares creadas con propósitos de bien común -cual es el fomentar el progreso de la zona o barrio donde vive y actúa- ha sido especialmente considerada y alentada en todo momento, por entender

4 El período de entreguerras es, quizá, el que mayor atención recibió de los historiadores de la ciudad. Pueden consultarse los trabajos de De Privitellio (2004), Walter (1993), Korn y Romero (2006); Korn (1974); Gutiérrez y Romero (1995). 
que esta clase de entidades -cuando no exceden su objetivo específico- constituye un valioso elemento de colaboración y utilísimo expediente para llegar al conocimiento directo de las necesidades y los deseos de los vecinos (Memoria Municipal 1933-1934, pp. 305-306).

Y en la de 1935, en la que se afirmaba que:

La acción desarrollada, el cúmulo de mejoras efectivas incorporadas a los barrios y la firme decisión del D.E. [Departamento Ejecutivo] fomentando la creación de estas entidades -reconociéndoles categoría representativa y propósitos de bien común- han contribuido para que en la actualidad no quede barrio que carezca de esa entidad eminentemente popular (Memoria Municipal 1935, pp. 366-367).

Como vemos, las sociedades de fomento suponen un modo específico de estructurar la institucionalidad local. El punto crucial para entender esta modalidad es la noción de reconocimiento, un mecanismo esencial en la construcción de la autoridad estatal, que permite, a través de un acto administrativo, instituir la representación de determinado grupo o asociación (Landau, 2008a; Frederic, 2004). El reconocimiento le permite al Estado actuar sobre la sociedad, apelando al mismo tiempo a su necesaria diferencia con los poderes públicos. Es, en este sentido, un dispositivo que actúa en la frontera, en el límite entre lo estatal y lo no estatal. Lo que constituye su razón de ser es el reconocimiento de la representatividad de determinada institución, a la que se considera habilitada para cumplir determinados fines. A través del reconocimiento de los representantes de las sociedades de fomento se constituye una figura que es central en el gobierno de los barrios: la del vecino gestor. Esta categoría supone la existencia de determinados individuos que, a través de ser investidos y reconocidos como representantes de la sociedad de fomento y, a través de ella, del barrio, se instituyen en los mediadores entre la comunidad de pertenencia y los poderes públicos.

La figura del vecino gestor supone una forma particular de pensar la relación entre el barrio y los poderes públicos: la participación debía hacerse en la escala barrial, y suponía simplemente la conformación de la demanda que debía realizarse frente a la Intendencia o al Concejo Deliberante. Se mantenía, sin embargo, una demarcación clara entre lo relativo al Estado municipal y lo concerniente a la comunidad barrial. Los vecinos gestores operaban como intermediarios entre el barrio y los poderes públicos. En este sentido, el gobierno de los barrios inauguró un discurso que, a partir de entonces, constituyó un lugar común en la gestión de los asuntos municipales: la crítica a la burocratización (el "expedienteo", según la fórmula más habitual de la época) de la Municipalidad y la valoración de la acción "directa", "cercana" y "personal" de los funcionarios comunales.

Así, durante la década de los treinta era frecuente que los funcionarios realizaran giras por los "barrios", con el fin de conectarse directamente con las problemáticas de los vecinos, que se transmitían a través de sus representantes de las sociedades de fomento. En relación con estas giras, en la Memoria Municipal de 1933-1934 se decía que "la explicación directa y personal que en cada caso puede hacerse ante los representantes del vecindario afectado o ante el vecindario mismo en el propio lugar, alcanza un poder de convicción que no lo consigue la fría resolución administrativa” (p. 306). 
Es importante diferenciar entre este gobierno de los barrios, y la descentralización administrativa o el gobierno descentralizado. La descentralización había sido un tópico recurrente del derecho administrativo y de la discusión política hacia fines del siglo XIX. Pero el gobierno de los barrios no es un gobierno descentralizado. Es un modo de estructurar el vínculo directo entre los vecinos y el poder central, en donde toda lógica de intermediación es vista como problemática. Se puede plantear, como hipótesis, que es justamente la imposición de este modelo, y su temprano éxito, lo que constituyó una forma de pensar el gobierno de la ciudad que impidió el desarrollo de un tipo de gobierno descentralizado.

\section{Buenos Aires durante el peronismo}

La institucionalidad local cambiará en Buenos Aires durante el peronismo ${ }^{5}$ En cuanto a las transformaciones urbanas, por entonces Buenos Aires se convirtió definitivamente en una metrópoli que se extendía más allá de sus demarcaciones jurisdiccionales. En lo relativo a las concepciones de las elites gubernamentales, para el peronismo las ciudades argentinas en general, y Buenos Aires en particular, no podían ser concebidas como organismos independientes. Por el contrario, debían ser consideradas como partes constitutivas de una organización más amplia, de nivel nacional. En virtud de ello, el gobierno de la Capital Federal debía establecerse a partir de la lógica de la delegación que hicieran el presidente de la Nación y el Congreso en ejecutantes locales, sin perder no obstante su potestad sobre la ciudad. Esta particular manera de organizar el gobierno de la ciudad supuso a la vez una continuidad de las concepciones científico-técnicas del gobierno de la ciudad, aunque enmarcadas en un relato nacional. El leitmotiv del peronismo fue la necesidad de contar con la planificación y el planeamiento que permitieran articular la resolución de problemas locales con las metas de la nación toda. (Sobre este punto, véase Jajamovich, 2007).

El proceso de nacionalización del gobierno científico-técnico de la ciudad llevó a una modificación de la institucionalidad local. La concepción peronista del gobierno de la ciudad planteaba una relación directa entre el ámbito local y los poderes nacionales. Si la única referencia es la nacional, la lógica barrial pierde toda su razón de ser. En este contexto, la figura del vecino gestor, desarrollada en los años de entreguerras, dejó de tener vitalidad, ya que se había construido sobre la lógica de una representación particularista y fragmentaria. En tanto que los representantes de las sociedades de fomento se presentaban como la encarnación del interés del barrio, tenían en este su razón de ser y su límite. La lógica impuesta por ese modelo se basaba en la total fragmentación del espacio de la ciudad, y con él el espacio de la representación.

No hay que ver, no obstante, en la eliminación de la comunidad barrial y el vecino gestor una supresión sin más de toda participación en los lugares de residencia, sino

5 A diferencia de lo que ocurre con el período de entreguerras, son más escasos los trabajos que se ocupan del período peronista en la ciudad. Diversos aspectos ligados a las características generales que adoptaron los gobiernos municipales durante el peronismo, y en especial el de la Ciudad de Buenos Aires, pueden consultarse en Macor (2004) y Marcilese (2009). Sobre el caso específico de la Ciudad de Buenos Aires, véase Aboy (2005), Acha (2004), Berman (2009, 2010), Landau (2013), Pírez (1999). 
un cambio en el modo en que se la concebía y canalizaba institucionalmente. En algunos casos, ello se dio a través de la transformación de las formas que adquiere la vida asociativa. Pero no es ello lo que constituyó el núcleo de la transformación: lo que cambió no fue el ámbito de la participación en sí, ni las actividades asociadas con ello, sino el marco de sentido en el que esas actividades se inscribían, que pasaron de referenciarse en la comunidad barrial, para comenzar a hacerlo en la totalidad nacional o, en los propios términos del lenguaje peronista, en la "comunidad organizada".

Este nuevo marco tuvo consecuencias para las sociedades de fomento, que hasta la llegada del peronismo constituían el modo privilegiado de institucionalidad local por parte de los poderes públicos municipales. Muchas de ellas desaparecieron o se politizaron. De hecho, muchas se proclamaron abiertamente peronistas, y fueron reconvertidas en unidades básicas (Romero \& De Privitellio, 2005). Sin embargo, no hay que comprender este movimiento como una total desaparición del espacio asociativo barrial en general y de las sociedades de fomento en particular. Aun en un nivel muy inferior al de años previos, durante las intendencias peronistas siguió la modalidad del reconocimiento de estas asociaciones, Perón recibió a sus delegados, y se desarrolló el Congreso de Sociedades de Fomento, auspiciado por el gobierno peronista. En los últimos días de su gobierno, en una reunión con los Vecinos de la Avenida San Martín, Perón dio muestras de una valoración de esta modalidad de participación. Al respecto decía:

Las comisiones de fomento son (...) los entes naturales y lógicos de colaboración con el gobierno municipal. (...) El adelanto, el desarrollo de las grandes ciudades ha creado problemas de gobierno extraordinariamente importantes. (...) El gobierno de Buenos Aires es cada vez un asunto más difícil y complicado. (...) La población y la extensión del Gran Buenos Aires nos están indicando la necesidad de una organización más adecuada a las actuales exigencias. (...) Ustedes están más adelantados que nosotros, que seguimos con un gobierno centralizado en la municipalidad cuando en realidad ha llegado el momento de ir descentralizando hacia los barrios el gobierno y la vida de la ciudad (Comisiones de Fomento con Perón, 1954, p. 5).

Esta valoración de la participación vecinal no se oponía, sin embargo, a una lógica centralizadora. Fue en los últimos años del peronismo, al darse un "acercamiento" a las sociedades de fomento, que se estableció una mayor concentración de funciones municipales en las oficinas nacionales. En este sentido, en 1954 y 1955 se sancionaron dos decretos a través de los cuales la relativa descentralización de la Intendencia se vio notablemente limitada, al hacerla depender del Ministerio de Interior.

\section{Buenos Aires luego de la caída del peronismo}

La inestabilidad política de los años que separan el golpe militar contra Perón en 1955, del inicio del gobierno de Alfonsín, en 1983, signados en buena medida por la proscripción del peronismo y los recurrentes golpes de Estado, hace difícil un análisis de las concepciones sobre el gobierno de la ciudad y la institucionalidad local. En lo concerniente al gobierno de la Capital Federal, a partir de la caída del peronismo se restableció el régimen municipal estipulado por la ley orgánica 
municipal reformada en 1917, en la que se había implementado el voto universal para concejales, que a partir de ese momento incorporaría por primera vez a las mujeres. Ello condujo al desarrollo, luego de una década de centralización peronista, de algunas de las temáticas "municipalistas": reimplantación de la rama deliberativa del gobierno municipal, elección directa de los concejales, discusión sobre la elección directa del intendente, etcétera.

Este redescubrimiento de lo municipal convivía con el desarrollo urbano incesante, que ahondó la problemática del "Conurbano"6. Para enfrentar esta cuestión, los saberes urbanísticos profundizaban el ideal científico-técnico, que planteaba la necesidad de establecer instituciones y acciones basadas en un conocimiento experto sobre esta cuestión, además de los planes reguladores, las comisiones de expertos, etcétera. En suma, la situación era paradójica, en tanto que se planteaba una vuelta a un gobierno municipal relativamente independiente del nacional, en momentos en que la realidad urbana marcaba una progresiva interdependencia de la ciudad respecto a los municipios aledańos, y a la nación toda.

Lo anterior tuvo consecuencias en lo relativo a la institucionalidad local, y varias fueron las innovaciones. La primera, desarrollada en algunos períodos dictatoriales, fue la instauración por decreto de las Juntas Representativas Vecinales en 1969, las cuales comenzaron a funcionar en 1970 y se extendieron hasta la convocatoria a las elecciones de 1973, para luego ser reimplantadas durante la dictadura de 1976-1983. En términos discursivos, debía estructurarse una Junta por barrio, y estar compuestas, como recuerda Del Brutto (1986), "por miembros que pertenecían a Asociaciones de Fomento, Clubes de Madres, parroquias, centros comerciales y/o profesionales, asociaciones de amigos de calles, avenidas, plazas o barrios" (p. 59). Sin embargo, debemos ser cautos al examinarlas, puesto que no es un dato anecdótico que este tipo de iniciativas se realizara en un marco de suspensión del Estado de derecho, y del que solo participaran aquellas personas que apoyaban al gobierno militar.

La segunda innovación, que constituye quizá uno de los datos más significativos, se dio en 1972, cuando el régimen militar sancionó una nueva ley orgánica de la ciudad, la 19987, que se puso en práctica en mayo de 1973, ya en el tercer gobierno peronista. Entre los cambios introducidos, se instauró un mecanismo electoral a escala local: los consejos vecinales. Según el artículo 44 de la ley, los consejos vecinales tenían, en particular, como funciones:

a) estimular la actividad cívica y la participación comunitaria; b) informar y asesorar respecto al estado y necesidades del vecindario, colaborando en la formulación de programas de interés comunal; c) proponer al Departamento Ejecutivo anteproyectos de obras, servicios y trabajos (...); h) realizar el contralor de gestión de los servicios (...) que se ejecuten dentro de su jurisdicción (Ley No 19987, Capítulo 11, art. 44). 
El dispositivo de los consejos vecinales supuso una novedad respecto a la figura del vecino gestor expresada en el modelo de las sociedades de fomento. En estas últimas, la actividad era concebida en términos civiles, pero no políticos, ya que esta categoría solo podía ser expresada por los concejales elegidos por el voto popular. Esta distinción se borra al hacer electivo el cargo de los consejeros vecinales. Si bien en la práctica estos consejos tuvieron una escasa influencia en el gobierno de la ciudad y pasaron inadvertidos a los ojos de la mayor parte de la población porteña, fueron sumamente significativos en cuanto a la emergencia de una nueva concepción de la relación entre los gobernantes y los gobernados. Ello es así, porque por primera vez un representante vecinal era elegido a través del mecanismo del voto universal.

El golpe de Estado de 1976 interrumpió la experiencia de los consejos vecinales. Los jefes de las tres fuerzas militares nacionales decidieron remover a todas las autoridades del país, incluyendo al intendente de Buenos Aires, y suspender toda actividad política y gremial. A los pocos días asumió el intendente de facto, Osvaldo Cacciatore. Poco tiempo después de su asunción, el Poder Ejecutivo le asignó las facultades que la ley les otorgara al Concejo Deliberante y a los consejos vecinales. Durante los años que duró el régimen militar, el gobierno de la ciudad siguió los lineamientos de una lógica tecnocrática y autoritaria.

\section{Buenos Aires desde el retorno democrático}

A partir del retorno de la democracia en 1983, comenzó un proceso cuyo común denominador era el afianzamiento de la idea según la cual era necesario modificar la manera en que se gobernaba la Ciudad de Buenos Aires. Durante los años ochenta, y con mayor fuerza a partir de la asunción de Menem en 1989, se llevó a cabo una reforma del Estado municipal que introdujo cambios en sus funciones, responsabilidades y atribuciones. La necesidad de reformar el gobierno de la ciudad contemplaba también a los consejos vecinales. Pese a que estas instituciones habían tenido una vida escueta y poco significativa entre 1973 y 1976, el retorno democrático las volvió a poner en primer plano. Como recuerda Del Brutto (1989, p. 93), en 1984 se diseñó en la Municipalidad una Subsecretaría de Consejos Vecinales que se planteaba articular las demandas territoriales en torno a la acción de los consejos. Sin embargo, dicha articulación no prosperó, transformándose en la Dirección de Relaciones con la Comunidad, que establecía un vínculo ajeno a la acción de los mismos.

Casi al mismo tiempo, en enero de 1984, el Concejo Deliberante trató un proyecto de ordenanza cuyo objetivo era pagar un "viático de movilidad" a los consejeros, que -según lo estipulaba la ley- debían cumplir sus tareas ad honorem. El tema del pago de las tareas del consejero no es una cuestión menor, puesto que se liga directamente con la profesionalización de la actividad, que sale del ámbito de la participación voluntaria para convertirse en una responsabilidad pública. En otras palabras, convierte a un participante en un funcionario, algo que era defendido por la mayoría de los concejales. 
Pero no era solo el Concejo Deliberante el que impulsaba esa medida. En septiembre de 1984, el diputado radical Liborio Pupillo presentó un proyecto en el Congreso Nacional, que fue sancionado en septiembre de 1985, en el que se planteaban diversas reformas a los consejos vecinales, entre las que destacaban la reforma de las zonas de influencia, para hacerlas coincidir con las circunscripciones electorales; el otorgamiento de fueros similares a los de los diputados; la remuneración de la tarea, etcétera. Sin embargo, la ley no fue promulgada por el presidente Alfonsín, que la devolvió al Congreso el 31 de octubre. Lo más destacado de este suceso es la justificación que hizo el presidente de su decisión. En palabras de Alfonsín, reproducidas en el Diario de Sesiones de la Cámara de Diputados de la Nación:

La comunicación entre las autoridades y vecinos, es decir, la posibilidad de que aquellos conozcan los problemas, las demandas y los intereses de estos, exige necesariamente la creación de mecanismos descentralizados a lo largo de la ciudad que sirvan de puente de conexión entre unos y otros. Estas razones son las que requieren la revisión y modificación de las normas para regular el funcionamiento de los consejos vecinales de modo que puedan cumplir más acabadamente con su función. El proyecto de ley 23303 modifica algunos aspectos de las reglas aplicables a los consejos vecinales, pero sin satisfacer correctamente los principios de promoción de la participación popular como un compromiso cívico y la descentralización del poder como un objetivo inherente al sistema democrático. El consejo vecinal debe ser un ámbito de comunicación y acción de los vecinos de la Capital Federal, una institución política básica de participación en el gobierno comunal, pero de ningún modo un organismo municipal más. Las normas de los incisos 2, 8, 11 y 12 del artículo 1 hacen que el cargo de consejero tenga las características más que de un representante vecinal, de un funcionario público. (...) Esta deformación del perfil que debería tener el consejero vecinal se refleja también al determinar en forma general para consejero vecinal pautas de organización que deberían surgir espontáneamente de la comunidad a través de la participación desarrollada en su ámbito natural, para luego sí, de ser necesario, otorgarles la regulación normativa que corresponda para su institucionalización. (...) Por las razones expuestas, se devuelve a vuestra honorabilidad el proyecto de ley 23303, sin promulgar (p. 7219).

La cita no podría ser más elocuente, puesto que muestra que lo que se discutía era la naturaleza misma de la institución, que para Alfonsín se veía desvirtuada al deformarse el perfil que debería tener el consejero. Este no podía de ningún modo ser considerado un funcionario público, sino simplemente un representante vecinal que operaría como puente entre las demandas de los vecinos y las autoridades municipales. En otras palabras, no sería más que un modo de canalización institucionalizada de las demandas de los vecinos, un nexo que, sin embargo, no debía conducir a asociarlo con un ente gubernamental. Lo que muestra esta discusión es la ambivalencia misma que constituía a los consejos vecinales. Luego de este incidente, los consejos vecinales continuaron ejerciendo sus tareas de manera precaria, y más allá de algún pedido puntual de informes sobre su situación, fueron quedando en el olvido, hasta su desaparición. 
En paralelo a la progresiva agonía de los consejos vecinales, emergía una novedosa forma de concebir la institucionalidad local, que el discurso político y académico presentaba como si fuese una total novedad respecto al pasado. Su característica más significativa era la invocación del objetivo de sobrepasar los estrechos límites de la democracia representativa, para instaurar mecanismos participativos en los que los diversos actores involucrados, tanto estatales como no estatales, los gobernantes y los gobernados, se asociaran en prácticas de cogestión consensuadas. Desde la década de los ochenta, este nuevo discurso -que puede ser resumido en la famosa fórmula de la "participación ciudadana" - fue creciendo de la mano del planteo de la necesidad de reformar el Estado municipal y desarrollar formas de gestión menos burocráticas y más descentralizadas. Durante los ańos ochenta fueron varios los proyectos que se presentaron en el Parlamento en pos de incluir una mayor participación de los vecinos de la ciudad. En 1988, el diputado Canata presentó un proyecto de reforma de la ley orgánica que se concentraba en la inclusión de mecanismos de participación de democracia semidirecta, como la iniciativa popular, consulta o la revocatoria. Iniciativas similares presentaron otros diputados, como González Gass, Argüello, Dalesio de Viola, Varela Cid. Si bien muchos de ellos planteaban una reforma integral de la ley municipal, que incluía la elección directa del intendente o el cambio en el modo de elección de concejales, lo más significativo es que todos proclamaban la inclusión de procedimientos de participación.

Varias de las inquietudes emergentes de la década de los ochenta fueron retomadas con más fuerza en los noventa, en el contexto del proceso de transformación institucional del gobierno de la ciudad. En la Constitución Nacional de 1994, en su artículo 129, se establece que Buenos Aires "tendrá un régimen de gobierno autónomo con facultades propias de legislación y jurisdicción y su jefe de gobierno será elegido directamente por el pueblo de la ciudad". Luego del cambio constitucional, se sancionó, en 1996, la Constitución de la Ciudad Autónoma de Buenos Aires, que estipulaba la elección directa del titular de la rama ejecutiva, el Jefe de Gobierno, y de los legisladores de la ciudad, denominados de allí en más diputados de la ciudad. Además, preveía la instauración de una "democracia participativa” a través de la implementación de múltiples instancias de participación de los vecinos de la ciudad, y un programa descentralizador ${ }^{7}$.

Para llevar a cabo estos objetivos fueron eliminados los consejos vecinales, que experimentaban por entonces un gran desprestigio. En su lugar, la Constitución de la Ciudad Autónoma de Buenos Aires estipulaba la conformación de comunas, que serían unidades de gestión política y administrativa de escala local, cuyas autoridades serían elegidas por los vecinos de cada una de ellas. Sin embargo, su puesta en funcionamiento fue reiteradamente retrasada y en su lugar se crearon los Centros de Gestión y Participación (CGP), que eran unidades administrativas descentralizadas a cargo de funcionarios nombrados por el Jefe de Gobierno de la ciudad. A ello se le sumó la implementación de diversos programas públicos participativos, bajo el imperativo común de acercar el gobierno a los ciudadanos. Finalmente, la Ley de

7 Hemos desarrollado un análisis del proceso de autonomización del gobierno de la ciudad y la implementación de políticas públicas participativas en Landau (2008c), y en la última parte de Landau (2010). Remitimos al lector a estos textos para profundizar sobre este punto. 
Comunas se sancionó en 2005. La misma estipula que las comunas estarían gobernadas por un órgano colegiado de siete miembros, llamado Junta Comunal, elegido a través del voto popular de los vecinos de cada comuna. La tarea de los comuneros es remunerada con el 60 por ciento del ingreso de un diputado de la ciudad. En cuanto a las funciones, la ley estipula algunas tareas exclusivas y concurrentes. Pese a la existencia de la ley, las primeras elecciones recién se llevaron a cabo en 2011. En la actualidad, cada comuna cuenta con un presupuesto anual que debe ser consensuado con los vecinos de manera participativa. En cada una debe funcionar, además, un Consejo Consultivo constituido por las asociaciones vecinales y partidos políticos de la zona. Con todo ello se abre una nueva etapa en cuanto a la institucionalidad local de la ciudad.

\section{La institucionalidad local en Buenos Aires en perspectiva histórica}

El recorrido histórico realizado nos estimula a preguntarnos por las continuidades y rupturas en lo relativo a la relación entre institucionalidad local y gobierno de la ciudad. Un común denominador que atraviesa el siglo que hemos analizado es la invocación a generar una colaboración entre autoridades públicas y habitantes como medio de acercar el Estado a los barrios. En 1895, como hemos visto, la Revista Municipal se quejaba de aquellos:

Que censuran y gritan a tort et à travers invocando a cada rato su título de contribuyente y su amor por el adelanto de la ciudad [pero que] nada hacen para cooperar a la acción municipal y facilitar su tarea.

Y proponía, ante esta situación, "la formación de sociedades parroquiales de fomento, que acostumbrando a los contribuyentes a ocuparse de las cosas e intereses municipales serían auxiliares de la autoridad y estímulo a sus esfuerzos" (Revista Municipal, 1985, p. 1606).

Ya entrado el siglo xx, en la Memoria Municipal de 1933-1934, se planteaba que a través de las giras que hacía el intendente o sus secretarios por los barrios, se había logrado mejorar el vínculo entre las autoridades municipales y los vecinos ya que:

La explicación directa y personal que en cada caso puede hacerse ante los representantes del vecindario afectado o ante el vecindario mismo en el propio lugar, alcanza un poder de convicción que no lo consigue la fría resolución administrativa (Memoria Municipal, 1933-1934, p. 306).

Más tarde, en 1955, Perón hacía referencia a que las sociedades de fomento son "los entes naturales y lógicos de colaboración con el gobierno municipal [y que] ha llegado el momento de ir descentralizando hacia los barrios el gobierno y la vida de la ciudad". Por último, la Ley 19987 estipulaba que los consejos vecinales tienen como una de sus misiones, la de "informar y asesorar respecto al estado y necesidades del vecindario, colaborando en la formulación de programas de interés comunal [y de] realizar el contralor de gestión de los servicios” (Capítulo 11, art. 44). 
Encontramos en estos antecedentes palabras que se asemejan a las de hoy: cooperación o colaboración con la acción municipal, fomento de construcción de entidades de bien común, necesidad de una descentralización, apoyo a la actividad de las asociaciones barriales, impulso al control de los poderes públicos. ¿Ello quiere decir que no habría ninguna diferencia entre esas formas de institucionalidad local y las actuales? No, esa no es la conclusión que nos parece adecuado plantear. Pero hay que desconfiar de aquellos discursos que presuponen una total novedad respecto al pasado.

Podemos distinguir esquemáticamente, siguiendo las cuatro citas antes mencionadas, cuatro momentos que permiten dibujar una relativa continuidad histórica. El primero es la emergencia de una valorización de la relación entre los habitantes de los vecindarios y las autoridades municipales, ligada a la conformación de asociaciones vecinales, que se alejaba por entonces de los principios económicos vinculados al voto censitario de la ciudad. A fines del siglo xix, este proceso abrió el camino a una concepción más abierta de la ciudad. El segundo es el reconocimiento estatal de las sociedades de fomento, instituido como política municipal en los años veinte y treinta. Por entonces, la Municipalidad de la Ciudad de Buenos Aires no se remitía, como antes, a incentivar o fomentar la participación, sino que constituía un dispositivo por el cual las sociedades de fomento ocupaban un rol fundamental, dado que eran investidas por el Estado, a través del reconocimiento de la representación del barrio, y a cambio tenían la obligación de constituirse en la mediación entre las demandas de los vecinos y las autoridades públicas. El tercer momento remite a la modificación que el peronismo introdujo en este esquema. La invocación a la descentralización suena rara en la boca de Perón, puesto que el peronismo se caracterizó por una nacionalización del gobierno de Buenos Aires. Sin embargo, hay que señalar que el peronismo no eliminó totalmente, como hemos dicho, la participación en los ámbitos de residencia, sino que cambió la referencia para pensarla y regularla. En esta perspectiva, la figura de la comunidad barrial tendió a erosionarse, puesto que la ciudad y todo lo que ocurre allí pasó a ser interpretado como una parte constitutiva de un organismo más amplio: la comunidad organizada. El cuarto y último momento corresponde al período de los consejos vecinales, instituciones de barrio en las que los vecinos designaban a sus representantes a través del voto. Como hemos visto anteriormente, los consejos fueron eliminados en el proceso de "autonomización" de la ciudad, con la excusa de su supuesta ineficacia.

Si analizamos el proceso en el largo plazo, podemos observar que, aunque no siempre de forma recta, se puede trazar una línea de continuidad entre fines del siglo xIx y fines del xx, constituida por una persistente estatización de la institucionalidad local. Primero, se abre la participación a todos los habitantes, criticándose los criterios restrictivos de los principios económicos vigentes hasta entonces. Luego, se la reconoce oficialmente, aunque manteniéndola por fuera del ámbito de la representación estatal, como lo muestra el caso de las sociedades de fomento. Por último, se la incorpora entre las autoridades elegidas a través del sufragio, como es el caso de los consejos vecinales. Pero luego, en lugar de fortalecer estos consejos con un mayor nivel de autarquía, se los elimina para sustituirlos por los Centros de Gestión y Participación (CGP). En paralelo, comenzó a desarrollarse una multiplicidad de 
mecanismos, como los consejos consultivos de distinta índole, los mecanismos de democracia "semidirecta", los programas al estilo del Presupuesto Participativo o el Plan de Prevención del Delito.

Es sobre este desplazamiento que debemos posar nuestra atención. Para ello, es necesario analizar la relación establecida entre institucionalidad local y gobierno. El proceso de estatización de la institucionalidad local reconocía una diferenciación muy clara entre los ciudadanos o los vecinos, por un lado, y las autoridades públicas, por el otro. Ello incluso para el caso de los consejos vecinales que, pese a su representación política, no eran considerados como autoridades públicas. De hecho, recordemos que durante los años ochenta, como hemos analizado antes, el Congreso de la Nación sancionó una ley con el fin de pagar a los consejeros, que Alfonsín no promulgó, argumentando que se "deformaba el perfil" que debería tener el consejero, haciendo que tuviera "las características más que de un representante vecinal, de un funcionario público" (Mensaje Presidencial, 1986; en Diario de Sesiones de la Cámara de Diputados de la Nación, 6 y 7 de marzo de 1986, p. 7219). La argumentación que hacía Alfonsín era completamente comprensible, puesto que se basaba en una concepción de las autoridades estatales según la cual debían diferenciarse claramente de los ciudadanos, puesto que estarían investidas de un saber de gobierno (técnico, administrativo, burocrático) y de la representación del interés general. Y es justamente esta diferenciación la que será modificada a partir de fines de los ochenta, transformando la relación entre institucionalidad local y gobierno.

El punto nodal para comprender los cambios se vincula con la puesta en cuestión de la autoridad estatal en tanto figura que encarna el saber técnico especializado que permitiría resolver los problemas de gobierno de la ciudad, así como con la impugnación de su pretensión de representación del interés general. Este proceso, que comenzó a partir de la década de los setenta y se profundizó en los noventa, excede obviamente a la Ciudad de Buenos Aires (Landau, 2008a). Sin embargo, en este último caso, supone el marco en el que se discute el modo en que debería encararse el gobierno de la ciudad a partir de las transformaciones operadas con el cambio institucional. El discurso más difundido partía de argumentar que los problemas de eficacia y de representación se deben a que los gobernantes se alejaron de los ciudadanos. Pero, como acabamos de ver, no era la primera vez que se invocaba esta necesidad de acercamiento. Ello nos conduce a preguntarnos por la particularidad propia del modo en que el discurso de la cercanía establece una relación específica entre gobernantes y gobernados en la actualidad.

A diferencia de lo ocurrido en el pasado, la relación de cercanía entre autoridades estatales y ciudadanos que se postula no remite simplemente a una cercanía física, sino a una proximidad ontológica entre los sujetos. Las experiencias previas que planteaban la necesidad de una acción estatal que se hiciera presente en los barrios, o que diera la posibilidad a los vecinos de elegir consejeros vecinales que fueran los intermediarios frente a los poderes públicos, mantenían una diferenciación clara entre los gobernantes y los gobernados, estipulando para cada uno de ellos un rol claramente definido. Lo que trajo como novedad el modo de institucionalidad local, que se profundizó con el proceso de reforma de los ochenta y de autonomía de los noventa, fue la puesta en discusión de esta separación, con un discurso que los 
tendía a presentar como actores en pie de igualdad, como pares que toman parte de un proceso de elaboración colectiva. Es por eso que los dispositivos más significativos son aquellos que, como los programas participativos o los consejos de diversa índole, postulan diversas formas de gestión asociada.

Si comparamos esta última situación con las examinadas en relación con el pasado, aparecen claras diferencias. Antes, la frontera entre las autoridades públicas y vecinos no podía ser franqueada. Aun si, por ejemplo, en los ańos treinta los funcionarios municipales hacían giras por los diferentes barrios con el fin de lograr un acercamiento entre ellos y la Municipalidad, el objetivo no era otro que encontrar los mecanismos para que los pedidos de los vecinos arribaran lo más rápidamente posible a los poderes públicos. No se pretendía cambiar el hecho de que ellos, los funcionarios municipales, serían los únicos responsables de la resolución de los problemas planteados, puesto que se percibían como los poseedores del saber experto y los representantes del interés general. Algo parecido puede plantearse en el caso de los consejos vecinales. Pero durante las últimas décadas del siglo xx se puso en discusión esta separación, constituyéndose una nueva manera de pensar la relación entre gobernantes y gobernados caracterizada por hacer más borrosos los límites que históricamente se planteaban entre ambos.

Esta interpretación ontológica, y no simplemente física o territorial, de las relaciones de cercanía se asocia a lo que en otro trabajo hemos denominado la "metáfora espacial" (Landau, 2008b). En este caso, la dicotomía cerca/lejos opera no solamente como par de categorías habilitantes de un análisis realmente espacial, sino que en muchos casos sustituye metafóricamente a otras dicotomías, como bueno/ malo, eficiente/ineficiente, representativo/no representativo. Y lo que se postula no es solo que los gobernantes se hagan presentes en los ámbitos más locales, como el barrio, sino que estén dispuestos a incluirlos en la toma de decisiones. Partiendo de la constatación de una pérdida de confianza de los ciudadanos en sus autoridades públicas, que sería consecuencia de un alejamiento, se postula que el acercamiento contribuiría a resolver los problemas ligados a la pérdida de confianza de los ciudadanos respecto de las autoridades públicas y a mejorar las acciones del gobierno. Pero, ¿cómo operar este acercamiento ontológico entre gobernantes y gobernados? Es aquí que aparecen diversas fórmulas, como la de "democracia participativa" y la de "democracia deliberativa" (Blondiaux \& Sintomer, 2002), aunque ninguna es tan significativa para nuestro análisis de la metáfora espacial como la fórmula adoptada por los franceses, que directamente hablan de "democracia de proximidad" (Le Bart \& Lefebvre, 2005). La edificación de la metáfora espacial encuentra en el ámbito urbano un lugar ideal para su desarrollo. En efecto, un medio esencialmente espacial como es el de las ciudades se constituye en el ámbito privilegiado para desarrollar aquellas categorías políticas que dan sustento a los modos de gestión participativos.

Surge, naturalmente, la pregunta por las perspectivas futuras, en un escenario marcado por la reciente puesta en funcionamiento de las comunas. Sin pretensión de hacer análisis prospectivos, nos interesa señalar, para cerrar el artículo, que el gran interrogante es qué pasará en torno a la tensión establecida entre la "cercanía física" y la "proximidad ontológica”. En algún sentido, como hemos visto, son opuestas. La primera se basa en la idea de una separación clara entre los gobernantes 
y los gobernados. La segunda, por el contrario, tiende a desdibujar los límites. Es claro que las comunas supondrán la construcción de una institucionalidad estatal a una escala distinta de la ciudad. Sin embargo, aún resta saber qué tipo de relación se instituirá entre los miembros elegidos de la junta comunal, las autoridades centrales y los vecinos de la comuna. De ello depende, en buena medida, la resignificación del espacio local, del barrio, y de la naturaleza misma de los gobernantes y los gobernados, las autoridades y los vecinos. La letra de la ley, por un lado, construye a los comuneros en funcionarios estatales pagos, que cumplen funciones, porque fueron elegidos por los habitantes del barrio, y como tales pueden y deben encarnar los intereses generales del barrio. Pero, a la vez, establece la necesidad de construcción de un Consejo Consultivo del que pueden participar todas las asociaciones vecinales con intereses en el barrio. Del modo en que se establezca esta relación, en buena medida, depende el modo en que las comunas se instituyan como un tipo de institucionalidad local novedosa respecto al pasado. El desafío es lograr congeniar la "cercanía física" con la "proximidad ontológica". oeure

\section{Referencias bibliográficas}

Aboy, R. (2005). Viviendas para el pueblo. Espacio urbano y sociabilidad en el barrio Los Perales, 1946-1955. Buenos Aires: Fondo de Cultura Económica.

Acha, O. (2004). Sociedad civil y sociedad política durante el peronismo. Desarrollo Económico, 44(174), 199-230. uRL estable: http://www.jstor.org/stable/3456036

Armus, D. (1999). La ciudad higiénica: tuberculosis y utopías en Buenos Aires. En M. Gutman \& T. Reese (Eds.), Buenos Aires 1910: el imaginario para una gran capital (pp. 97-110). Buenos Aires: Eudeba.

Badía, G. \& Pereyra, E. (Eds.). (2005). Aportes a la cuestión del gobierno en la Región Metropolitana de Buenos Aires. Buenos Aires: Ediciones Al Margen.

Berman, R. (2009, octubre). El gobierno municipal de la Ciudad de Buenos Aires bajo el primer peronismo: debates en torno a la normalización desde su tratamiento parlamentario (1946-1955). XII Jornadas Interescuelas. Simposio realizado en Departamentos de Historia, Facultad de Humanidades de la Universidad Nacional del Comahue, Bariloche, Argentina.

Berman, R. (2010, octubre). El peronismo y la Ciudad de Buenos Aires. Notas sobre el gobierno local (1943-1955). En M. Bartolucci (Mod.), V Jornadas de Historia Política "Las provincias en perspectiva comparada". Simposio realizado en Centro de Estudios Históricos, Facultad de Humanidades, Mar del Plata, Argentina. Disponible en http:// historiapolitica.com/datos/biblioteca/vj_berman.pdf

Blondiaux, L. \& Sintomer, Y. (2002). L’impératif délibératif. Politix, 15(57), 17-35. Disponible en http://bit.ly/PHsp7s

Bourdé, G. (1974). Urbanisation et immigration en Amérique latine, Buenos-Aires: XIXe et XXe siècles. París: Montaigne.

Caride, H. (1999). La idea de Conurbano Bonaerense, 1925-1947. Los Polvorines: Instituto del Conurbano, Universidad Nacional de General Sarmiento. 
Carranza, A. (1926). La cuestión capital de la República (Vols. 1-5). Buenos Aires: Talleres Gráficos Argentinos de L. J. Rosso.

Comisiones de Fomento con Perón. A los delegados les habló en el salón blanco. (1954). Revista Plumadas, 76, 5.

De Privitellio, L. (2004). Vecinos y ciudadanos, sociedad y politica en la Buenos Aires de entreguerras. Buenos Aires: Siglo Xxi Editores.

De Privitellio, L. (2006). Un gobierno reformado para una nueva ciudad: el debate de la ley municipal de 1917. En F. Korn \& L. A. Romero (Eds.), Buenos Aires/entreguerras. La callada transformación, 1914-1945 (pp. 83-123). Buenos Aires: Alianza. [Artículo disponible en http://bit.ly/15k3pug]

Del Brutto, B. (1986). Politica municipal y participación. Buenos Aires: Centro Editor de América Latina.

Del Brutto, B. (1989) Partidos políticos y gestión urbana en Capital Federal. Buenos Aires: Centro Editor de América Latina.

Frederic, S. (2004). Participación política y reconocimiento: paradojas de la "descentralización" de la gestión urbana en Buenos Aires. En M. Escolar, G. Badía \& S. Frederic (Eds.) Federalismo y descentralización en grandes ciudades. Buenos Aires en perspectiva comparada (pp.187-208). Buenos Aires: Prometeo.

Gorelik, A. (1994). La búsqueda del centro. Ideas y dimensiones del espacio público en la gestión urbana y en las polémicas sobre la ciudad: Buenos Aires, 1925-1936. Boletín del Instituto de Historia Americana Dr. Emilio Ravignani, 3(9), 41-73.

Gorelik, A. (2004). La grilla y el parque. Espacio público y cultura urbana en Buenos Aires, 18871936. Quilmes: Universidad de Quilmes.

Gorelik, A. \& Silvestre, G. (1991). Imágenes al Sur. Sobre las hipótesis de James Scobie para el desarrollo de Buenos Aires. Anales del Instituto de Arte Americano e Investigaciones Estéticas, 27-28 (1989-1991), 2-34.

Gutiérrez, L. \& Romero, J. L. (1995). Sectores populares, cultura y política. Buenos Aires en la entreguerra. Buenos Aires: Sudamericana.

Hardoy, E. \& Gutman, M. (1992). Buenos Aires, historia urbana del área metropolitana. Madrid: Mafre.

Jajamovich, G. (2007, septiembre). Elementos para una historia del urbanismo: del urbanismo a la planificación. En Cuartas jornadas de jóvenes investigadores del Instituto de Investigaciones Gino Germani. Simposio realizado en Facultad de Ciencias Sociales, Universidad de Buenos Aires, Buenos Aires, Argentina. Disponible en http://bit.ly/19J2Ihh

Jurado, M. (1935). La ley orgánica de la Ciudad de Buenos Aires. Antecedentes, discusión parlamentaria, reformas. Buenos Aires: Universidad de Buenos Aires.

Korn, F. (1974). Los huéspedes del 20. Buenos Aires: Sudamericana.

Korn, F. \& Romero, L. A. (Eds.). (2006). Buenos Aires/entreguerras. La callada transformación, 1914-1945. Buenos Aires: Alianza.

Landau, M. (2008a). Cuestión de ciudadanía, autoridad estatal y participación ciudadana. Revista Mexicana de Sociología, 70(1), 7-45. Disponible en http://www.revistas.unam. $\mathrm{mx} / \mathrm{index} \cdot \mathrm{php} / \mathrm{rms} /$ article/view/6122

Landau, M. (2008b). La participación en las políticas públicas y los límites de la metáfora espacial. Revista Politica y Cultura, 30, 67-89. Disponible en http://bit.ly/1apidOf 
Landau, M. (2008c). Politica y participación ciudadana en la Ciudad Autónoma de Buenos Aires. Buenos Aires: Miño y Dávila.

Landau, M. (2010). Socio-historia de la cuestión del gobierno de la ciudad. Buenos Aires, de la federalización a la autonomía (1880-1996) (Disertación doctoral no publicada). École des Hautes Études en Sciences Sociales (ehess)/Universidad de Buenos Aires (UBA), París/Buenos Aires.

Landau, M. (2011). Elites, instituciones municipales y espacio urbano en Buenos Aires (18801917). Revista Ciudades, 92, 2-8.

Landau, M. (2012). De la ciudad civil a la ciudad social: concepciones de gobierno en Buenos Aires (1880-1955). Revista Nuevo Mundo/Mundos Nuevos. Recurso en línea. doi: $10.4000 /$ nuevomundo. 63230

Landau, M. (2013). 'Boedo nada quiere ni necesita salvo a Perón': el gobierno de la Ciudad de Buenos Aires en el primer peronismo. En Revista Estudios Sociales, 44, 123 - 151.

Le Bart, C. \& Lefebvre, R. (2005). La proximité en politique. Usages, rhétoriques, pratiques. Rennes: Presses Universitaires de Rennes.

Macor, D. (2004). Dinámica política y tradición constitucional: la reforma de 1949 en la provincia de Santa Fe. Quinto Sol, 8, 51-72. Disponible en http://bit.ly/17Kfw9X

Marcilese, J. B. (2009). Estado provincial y municipios bonaerenses, una relación conflictiva en los ańos del primer peronismo. Anuario del Instituto de Historia Argentina, 9, 149-178. Disponible en http://dialnet.unirioja.es/servlet/articulo?codigo=3842666

Morelli, F. (2007). Orígenes y valores del municipalismo iberoamericano. Araucaria. Revista Iberoamericana de Filosofía, Politica y Humanidades, 9(18), 116-129. Disponible en http://www.redalyc.org/articulo.oa?id=28291806

Paiva, V. (2000). Medio ambiente urbano. La emergencia del concepto. Concepciones disciplinares y prácticas profesionales en Buenos Aires entre 1850 y 1915. Revista Área, 8, 65-78.

Pírez, P. (1996). La Ciudad de Buenos Aires: una cuestión federal. Revista Mexicana de Sociología, 58(3), 193-212. URL estable: http://www.jstor.org/stable/3540881

Pírez, P. (1998). Management of urban services in the city of Buenos Aires. Environment \& Urbanization, 10(2), 209-222. doi: 10.1177/095624789801000209

Pírez, P. (1999). Gestión de servicios y calidad urbana en la Ciudad de Buenos Aires. EURE, 25(76), 125-139. http://dx.doi.org/10.4067/S0250-71611999007600006

Pírez, P. (2005). Buenos Aires: ciudad metropolitana y gobernabilidad. Estudios Demográficos y Urbanos, 20(3), 423-447. Disponible en http://www.redalyc.org/articulo. oa?id=31220301

Romero, L. A. \& De Privitellio, L. (2005). Organizaciones de la sociedad civil, tradiciones cívicas y cultura política democrática: el caso de Buenos Aires, 1912-1976. Revista de Historia, 1(1). Disponible en http://historiapolitica.com/datos/biblioteca/privityromero.pdf

Ruiz M, I. (1986). La federalización de Buenos Aires. Buenos Aires: Hyspamérica.

Sábato, H. (2008). Buenos Aires en armas. La revolución de 1880. Buenos Aires: Siglo XXI Editores.

Scobie, J. (1977). Buenos Aires: del centro a los barrios. Buenos Aires: Solar-Hachette.

"Sociedades de fomento. Un ejemplo a imitar". Revista Municipal, 15 de junio de 1895. 
Tella, G. (1994). Política municipal y espacio urbano (Buenos Aires 1880-1910). Buenos Aires: Centro Editor de América Latina.

Ternavasio, M. (1991). Municipio y politica. Un vínculo histórico conflictivo (Disertación doctoral no publicada). Facultad Latinoamericana de Ciencias Sociales (Flacso), Buenos Aires.

Walter, R. (1993). Politics and urban growth in Buenos Aires: 1910-1942. Cambdrige, MA: Cambridge University Press.

\section{Documentos oficiales}

Ley No 19987. Ley Orgánica de la Municipalidad de la Ciudad de Buenos Aires. Boletín Oficial. Buenos Aires, 11 de noviembre 1972.

Memoria Municipal 1933-1934. Municipalidad de la Ciudad de Buenos Aires, Buenos Aires, 1935.

Memoria Municipal 1935. Municipalidad de la Ciudad de Buenos Aires, Buenos Aires, 1935.

Mensaje Presidencial del Dr. Raúl R. Alfonsín. (1986, 6 y 7 de marzo). Diario de Sesiones de la Cámara de Diputados de la Nación, 7219.

República de Argentina. Constitución de la Nación Argentina. Boletín Oficial. Santa Fe, 22 de agosto 1994. 